\title{
Cold-fission yields at effective excitation energies
}

\author{
V. Avrigeanu, A. Florescu, and A. Săndulescu \\ Institute of Physics and Nuclear Engineering, P.O. Box MG-6, 76900 Bucharest, Romania \\ W. Greiner \\ Institute for Theoretical Physics, Johann-Wolfgang-Goethe University, D-60054 Frankfurt am Main, Germany \\ (Received 3 May 1995)

\begin{abstract}
The experimental cold-fission yields for the system ${ }^{233} \mathrm{U}\left(n_{t h}, f\right)$ are analyzed as function of the effective total excitation energy (TXE). The nuclear level density effect is taken into account at higher TXE, in order to benefit by the lower experimental data uncertainty as well as to avoid the quantitative account of the level densities close to fragment ground states. In this way the odd-even staggering which appears in the yields extrapolated at zero excitation energy by using the level densities, vanishes. We conclude that the cold nuclear fragmentation theory including the dynamical model describes well the experimental data.
\end{abstract}

PACS number(s): 25.85.Ec, 23.70. +j, 21.10.Ma

\section{INTRODUCTION}

In the true cold fission the final-fragments total kinetic energy (TKE) approaches the $Q$ value of the corresponding fragmentation, completely analogous with the cluster radioactivity [1]. Only recently it was possible to resolve the masses and nuclear charges one by one at very high TKE. An attempt to obtain yields for the cold fission into the ground states of the two fragments belongs to Schwab et al. [2]; the cold-fission yields of ${ }^{233} \mathrm{U}\left(n_{t h}, f\right)$, measured as a function of the total excitation energy $\mathrm{TXE}=Q-\mathrm{TKE}$, were divided by calculated level densities for the two fragment system and extrapolated to $\mathrm{TXE}=0$. An alternative analysis based on the effective excitation energy concept $[3,4]$ is given hereafter, and the results are compared with the nuclear fragmentation theory [5-7].

The real cold fission has been described as a cold rearrangement process with fragments in their ground states [5], similarly to the exotic decays. Already earlier we have shown [6] that with a single set of fragment deformations, containing the few experimental deformation values and the theoretically predicted values of Möller and Nix [8] slightly modified, it has been possible to reproduce the experimental charge distribution for all mass fragmentations and for all currently measured nuclei. Moreover, at lower kinetic energies we have assumed the excited deformed fragments additionally $\beta$ deformed [7]. Cold-fission isotopic yields of the thermal neutron induced fission of ${ }^{233} \mathrm{U}$ and ${ }^{235} \mathrm{U}$ have been calculated, e.g., for $\mathrm{TXE}=3 \mathrm{MeV}$ [9].

In order to see how barrier penetrabilities calculated under different assumptions for the fragment ground-state deformations may explain the experimental cold-fission yields, Schwab et al. have considered the effect of the nuclear level density of the noninteracting two fragment system and deduced the yield per energy level. Finally they also have analyzed the isotopic yields in the light-fragment mass range 76 $\leqslant A_{L} \leqslant 93$ at a fixed TXE, and explained the different behavior with respect to the ground-state yield distribution as being due to the influence of the level density. However, one should consider this influence at higher TXE values, to take advantage of lower experimental data uncertainty, and to avoid the quantitative account of the level densities close to

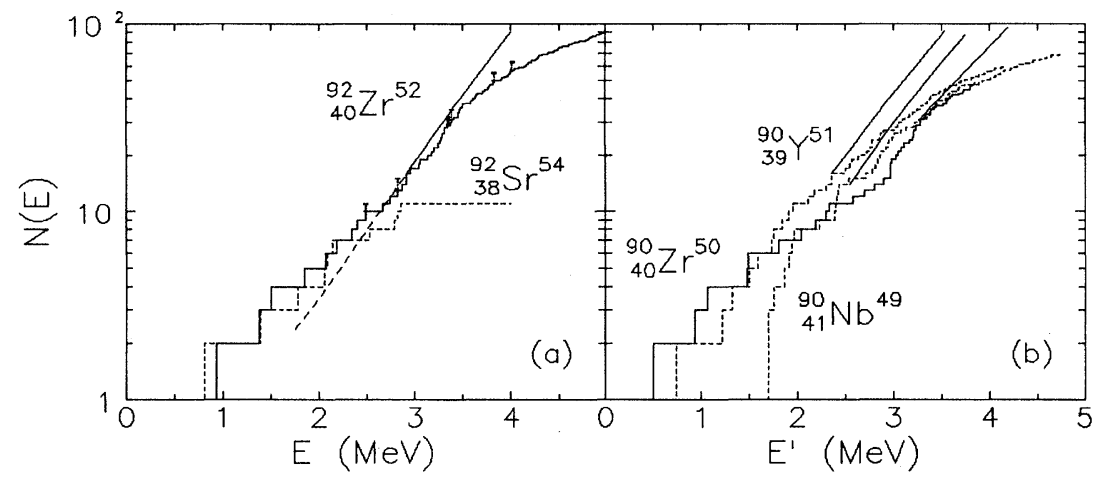

FIG. 1. Total number of low-lying discrete levels, experimental (histogram) and the BSFG-model description (full curves above the highest level fitted) for (a) the nuclei ${ }^{92} \mathrm{Sr}$ and ${ }^{92} \mathrm{Zr}$ versus excitation energy, and (b) ${ }^{90} \mathrm{Y}$, ${ }^{90} \mathrm{Zr}$, and ${ }^{90} \mathrm{Nb}$ versus the effective excitation energy. The BSFG parameters obtained for ${ }^{92} \mathrm{Zr}$ have been used for ${ }^{92} \mathrm{Sr}$ too, in order to check the parameter extrapolation to fissionfragments nuclei. 
TABLE I. Discrete levels number $N_{d}$ up to $E_{d}$ excitation energy, and $s$-wave neutron-resonance spacings $\bar{D}_{\text {exp }}$ in the neutron energy range $\Delta E[17]$ above the neutron binding energy $B_{n}$ [18], which have been fitted to obtain the BSFG-model level density parameter $a$ and shift $\Delta$ (corresponding to a spin cutoff factor calculated with the nuclear moment of inertia of a rigid body and a reduced radius $r_{0}=1.25 \mathrm{fm}$ ) for nuclei with $A \simeq 90$.

\begin{tabular}{lcccccc}
\hline \hline Nucleus & $N_{d}$ & $\begin{array}{c}E_{d} \\
(\mathrm{MeV})\end{array}$ & $\begin{array}{c}B_{n}+\Delta E / 2 \\
(\mathrm{MeV})\end{array}$ & $\begin{array}{c}\bar{D}_{\exp } \\
(\mathrm{keV})\end{array}$ & $\begin{array}{c}a \\
\left(\mathrm{MeV}^{-1}\right)\end{array}$ & $\begin{array}{c}\Delta \\
(\mathrm{MeV})\end{array}$ \\
\hline${ }^{90} \mathrm{Y}$ & 16 & $1.815^{\mathrm{a}}$ & 6.872 & $4.0 \pm 0.4$ & 9.50 & -0.54 \\
${ }^{90} \mathrm{Zr}$ & 31 & $4.541^{\mathrm{a}}$ & 11.972 & & 8.20 & 1.26 \\
${ }^{90} \mathrm{Nb}$ & 15 & $0.958^{\mathrm{a}}$ & 10.108 & & 9.00 & -1.58 \\
${ }^{92} \mathrm{Sr}$ & $(11)$ & $\left(2.850^{\mathrm{b}}\right)$ & 7.366 & & & \\
${ }^{92} \mathrm{Zr}$ & 16 & $2.909^{\mathrm{b}}$ & 8.645 & $0.57 \pm 0.10$ & 10.30 & 0.76 \\
\hline \hline
\end{tabular}

${ }^{a}$ Reference [19]

${ }^{\mathrm{b}}$ Reference [20]

the fragment ground states. This aim has been reached in the present work by analyzing the cold-fission yields at effective excitation energies.

\section{EFFECTIVE TOTAL EXCITATION ENERGY}

Within the worldwide used back-shifted Fermi gas (BSFG) level density model $[10,11]$ the effective excitation energy $E^{\prime}=E-\Delta$ is determined by the shift $\Delta$ of the ground state. This shift to a fictive ground state accounts for the conventional pairing energy, showing a very marked oddeven effect, as well as for the nuclear shell effects. Actually, the two main BSFG-model free parameters-the level density parameter $a$ and the ground-state shift $\Delta$-are determined by fitting the low-lying levels (in the limit that only few levels are missing within the present knowledge) and the average $s$-wave neutron resonance spacings. When the latter type of experimental data is not available for a given nucleus, one may use the method of a smooth curve for the level density parameter $a$ through the values deduced properly for the rest of nuclei in the respective mass region $[12,13]$, and obtain finally the $\Delta$-value parameter by fitting the discrete-level cumulative numbers. Examples of BSFG parameters obtained by using recent experimental data, for nuclei around $A \simeq 90$ (Fig. 1) are given in Table I. Grossjean and Feldmeier [14] have shown by means of the microscopic BCS-model calculation that the level density has different forms below and, respectively, above the phase-transition point where all nucleon pairs are broken. However, they showed also that, for a real nucleus with a finite number of particles and a discrete single particle spectrum, this discontinuity is smooth and well approximated by the BSFG model. Their algebraic formulas, obtained by inverse Laplace transformation of the grand canonical partition function for a Fermi gas with pairing interactions (but neglecting
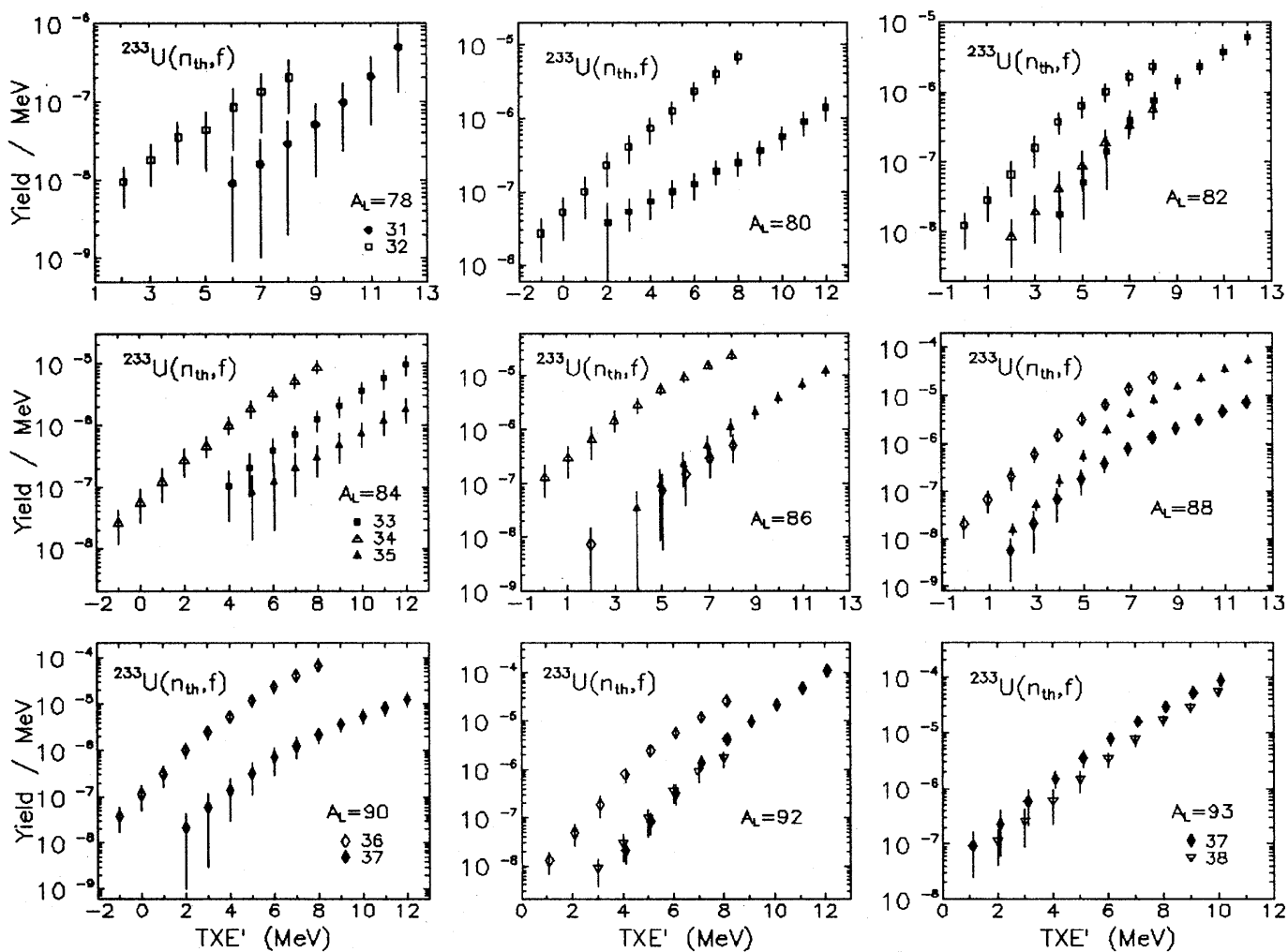

FIG. 2. Cold-fission yields per $\mathrm{MeV}$ of ${ }^{233} \mathrm{U}\left(n_{t h}, f\right)$ [2] as a function of the effective total excitation energy, for the even-even and odd-odd fragmentations with $78 \leqslant A_{L} \leqslant 92$ and the sample $A_{L}=93$ for even-odd and odd-even fragmentations. One nuclear charge is indicated by the same symbol in all cases. 


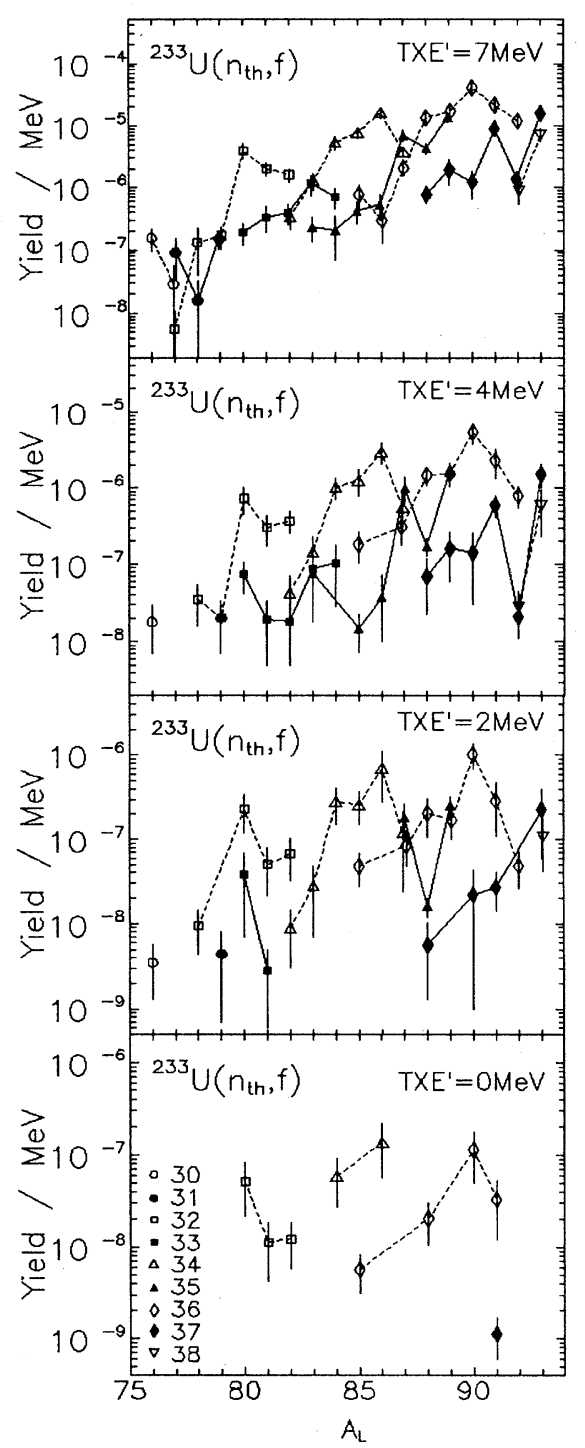

FIG. 3. Cold-fission isotopic yields per $\mathrm{MeV}$ of ${ }^{233} \mathrm{U}\left(n_{t h}, f\right)$ [2] at fixed effective total excitation energies of $0,2,4$, and $7 \mathrm{MeV}$. Different nuclear charges are indicated by different symbols. Full lines interconnect the yields for each odd- $Z$ charge number, while the dashed ones correspond to the even- $Z$ fission fragments.

the shell effects), contain two free parameters which are quite similar to the BSFG ones.

The systematic analysis along the Periodic Table [11] has shown that the ground-state shift $\Delta$ can be considered for $A>100$ as the difference between the pairing energy and a rather constant term equal to $\approx 1.5 \mathrm{MeV}$ on the average ("back shift"), with $\approx 0.5 \mathrm{MeV}$ fluctuations for individual nuclei; it is increasing as the inverse of the mass number for lighter nuclei. Thus $\Delta$-global values in the mass range $40<A<250$ could be taken around 1,0 , and $-1 \mathrm{MeV}$ for the even-even, odd- $A$, and odd-odd nuclei, respectively (see Fig. 2 of Ref. [11]), while the fitted particular values given in Table I may show the variance even in the limit of a magic shell region. The meaning of the effective excitation energy



FIG. 4. Potential-barrier penetrabilities for ${ }^{233} \mathrm{U}\left(n_{t h}, f\right)$ with both fragments in the ground states, versus the light-fragment mass number. The same symbols and lines as in Fig. 3 are used.

is depicted in Fig. 1(b) for this less favorable case. Although equal level densities in odd-odd and even-even nuclei are separated by $\simeq 3 \mathrm{MeV}$ of real excitation energy, they are rather well confined within $\sim 0.2 \mathrm{MeV}$ effective excitation.

Therefore, under the assumption of determinant level density effects, we choose to analyze the cold-fission yields corresponding to the same effective total excitation energy $\mathrm{TXE}^{\prime}=\mathrm{TXE}-\Delta_{L}-\Delta_{H}$, i.e., to similar level densities of the two fragment system for different fragmentations. Due to the lack of any parameter systematics for the fission fragments, we have used the above-mentioned global values of the shift parameter $\Delta$, which is a limitation of present analysis. However, it could be also mentioned that $\Delta$-value errors have even a stronger impact on the level densities used to obtain extrapolated ground-state yields.

\section{TXE' DEPENDENCE OF THE COLD-FISSION ISOTOPIC YIELDS}

The dependence of the experimental cold-fission yields [2] for the system ${ }^{233} \mathrm{U}\left(n_{t h}, f\right)$ on $\mathrm{TXE}^{\prime}$ (Fig. 2) proves a rather general ordering according to the odd-even nature of a given mass split fragmentation. The even-even fragmentation yields are definitely higher than the corresponding odd-odd ones at the same effective excitation energy, in contrast to the picture of the measured yields at a fixed TXE. This has been also the first element displayed by the pattern of the derived ground-state yields [2]. The shifts responsible for this result in our analysis are not present in the case of the odd- $A$ fragments, as it may be seen for the sample shown for $A=93$ in Fig. 2.

On the other hand, the isotopic yields show a similar behavior at fixed $\mathrm{TXE}^{\prime}$ ranging from 0 to $7 \mathrm{MeV}$ (Fig. 3). However, the picture becomes better defined at higher excitation, both in completion and increasing experimental accuracy. The rather parallel trend of the experimental coldfission yields versus $\mathrm{TXE}^{\prime}$ for the most of the mass splits (e.g., Fig. 2) has suggested already this conclusion, as well as the possibility to compare the theoretical prediction with the best experimental result depicted at $\mathrm{TXE}^{\prime}=7 \mathrm{MeV}$.

Following previous work $[7,9]$ we have estimated the penetrability $P(A, Z)$ through the potential barrier in the frame of the WKB approximation. The barrier was taken as 


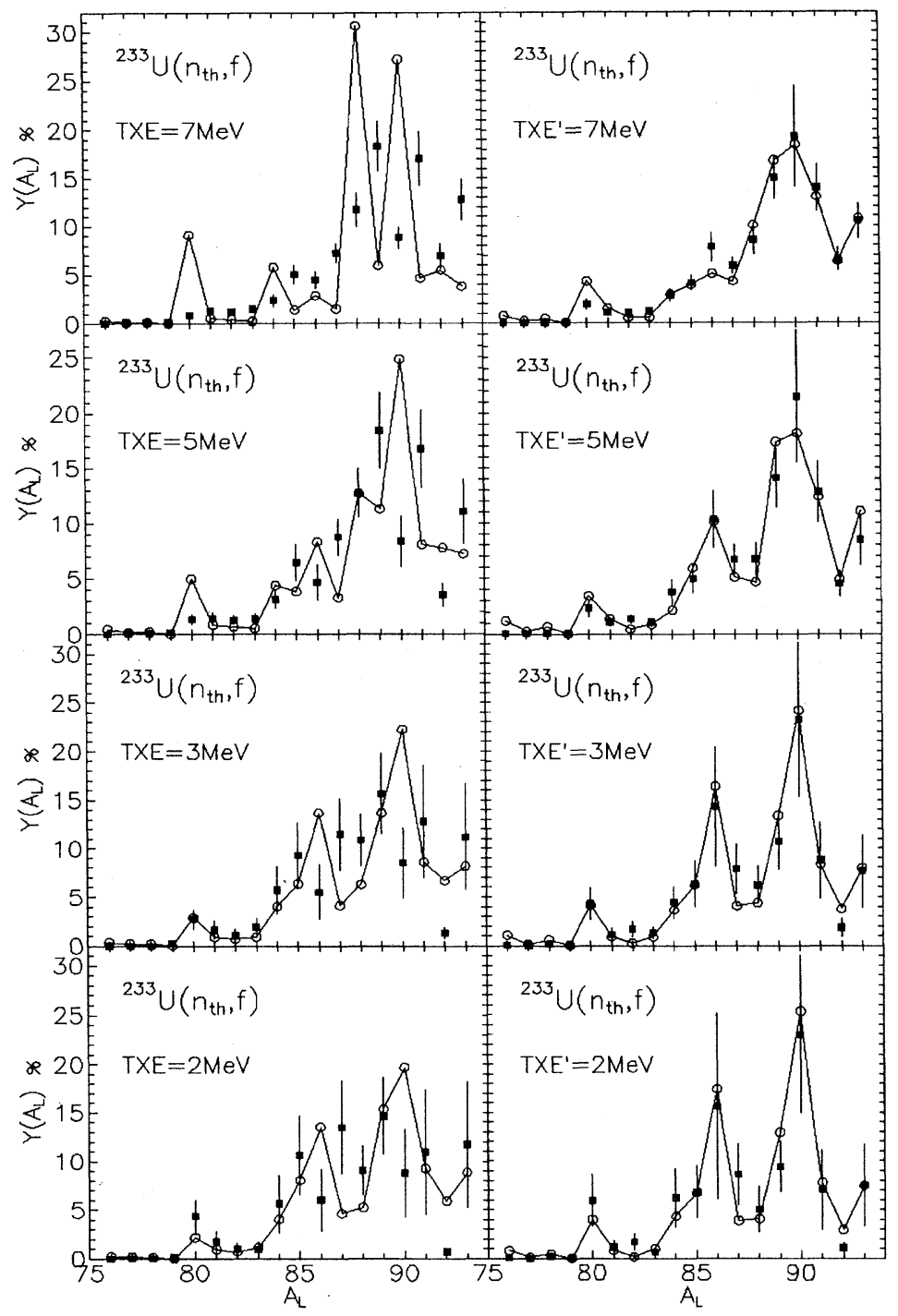

FIG. 5. Comparison of the calculated (open symbols) and experimental (full symbols) [2] cold-fission mass yields of ${ }^{233} \mathrm{U}\left(n_{t h}, f\right)$ for the light fragments $76 \leqslant A_{L} \leqslant 93$, at fixed TXE (left) and effective TXE (right) of 2,3,5, and $7 \mathrm{MeV}$. The yields are normalized to $100 \%$.

the sum between the nuclear proximity potential and the Coulomb potential for two deformed, coaxially aligned fragments. We assumed that the fragment TXE leads mainly to $\beta$-stretched fragments $[7,9]$ with final quadrupole deformations $\beta(E)=\left\{\beta_{0}^{2}+\left(\hbar^{2} / 2 B f E_{\beta}\right)\left[\left(2 E / f E_{\beta}\right)+1\right]\right\}^{1 / 2}$. Here $\beta_{0}$ is the static ground-state deformation, $B=\left(k / \epsilon_{2}\right) B_{2}(L D)$ is the vibrational mass parameter, $E_{\beta}=k^{\prime} / \epsilon_{2}$ is the $\beta$-phonon energy of the fragment and $0.9<f<1$ for the odd and oddodd splittings, corresponding to slightly larger deformations for TXE $<3 \mathrm{MeV}$. The parameters $k$ and $k^{\prime}$ have the same values as in $[7,9]$. This model is supported by recent experimental data, obtained by using triple $\gamma$-coincidence technique, which indicate that only the low-spin excited states are populated in the cold fragmentation process [15]. Finally, the isotopic yields $Y\left(A_{L}, Z_{L}\right)$ have been calculated as $Y\left(A_{L}, Z_{L}\right)=P\left(A_{L}, Z_{L}\right) / \Sigma_{A_{L} Z_{L}} P\left(A_{L}, Z_{L}\right)$.

The first step of the present comparison between the theo- retical and experimental cold-fission yields is making use of the barrier penetrabilities with both fragments in the ground states (Fig. 4). This resembles the analysis performed by Schwab et al. but includes zero-point $\beta$ vibrations. We have compared also these penetrabilities with the above-described isotopic yields at fixed TXE' values provided that the respective yields correspond to similar level numbers of the two fragment system. The trend of the isotopic-chain penetrabilities is closely related to the data while there is no odd-even staggering which has been found in the extrapolated yields at $\mathrm{TXE}=0 \mathrm{MeV}[2]$.

\section{COLD-FISSION MASS YIELDS AT FIXED TXE'}

A fully consistent analysis involves the total relative yield for a light mass fragment, $Y\left(A_{L}\right)=\Sigma_{Z_{L}} P\left(A_{L}, Z_{L}\right) /$ $\Sigma_{A_{L} Z_{L}} P\left(A_{L}, Z_{L}\right)$. These mass yields have been derived from 
the rich experimental data base of Schwab et al. and are calculated at four different $\mathrm{TXE}^{\prime}$ between 2 and $7 \mathrm{MeV}$. Actually, the value $\mathrm{TXE}^{\prime}=2 \mathrm{MeV}$ is the lowest effective total excitation energy still possible to be defined by using the present global values of the BSFG ground-state shift $\Delta$. The comparison of the experimental and calculated relative yields is shown in Fig. 5 when this analysis is not at fixed TXE but for similar TXE' values. Any level density effect on the experimental data is correctly reduced only in the latter case. Both the gross and the fine structure of the data are rather well reproduced by the fragmentation theory. The degree of the theory validation is increased by the use of more accurate experimental data available at higher TXE'. The lack of agreement for few less significant mass yields is better evidenced especially at $\mathrm{TXE}^{\prime}=7 \mathrm{MeV}$, and could be due to particular $\Delta$ values away from the global values.

\section{CONCLUSIONS}

Finally, we have found that the dynamical model of cold nuclear fragmentation describes well the yields of

${ }^{233} \mathrm{U}\left(n_{t h}, f\right)$ at different effective TXE. The present method takes into account the influence of the level density, avoiding the ambiguities at low excitation energies and making use of more accurate data at higher TXE. The odd-even staggering which appears in the extrapolated ground-state yields are not present anyhow in our analysis. This probably is due to underestimation of the level density close to the ground states for the even-even fragments in Ref. [2]. Actually, a systematics of the level density parameters for the fission fragments is of real interest for the present status of the cold-fission study. At the lowest excitation energies, the discrete levels should be used. The recent results obtained by using triple $\gamma$-coincidence technique [16] give total isotopic yields of the order of $10^{-4}$ per fission event or larger, due to the fact that they are integrated yields, while in TKE measurements they are differential and consequently much smaller. New experiments are necessary in which the $\gamma$ yields are measured in coincidence also with fission fragments of determined TKE.

\section{ACKNOWLEDGMENTS}

This work has been supported partially by the Romanian Ministry of Research and Technology under the Contract No. 150B. Help of T. Glodariu, Bucharest University Physics Department, in the data analysis is acknowledged.
[1] A. Săndulescu and W. Greiner, Rep. Prog. Phys. 55, 1423 (1992).

[2] W. Schwab, H.-G. Clerc, M. Mutterer, J.P. Theobald, and H. Faust, Nucl. Phys. A577, 674 (1994).

[3] T. Ericson, Adv. Phys. 9, 425 (1960).

[4] E. Gadioli and P.E. Hodgson, Pre-Equilibrium Nuclear Reactions (Clarendon Press, Oxford, 1992), p. 73.

[5] A. Săndulescu, J. Phys. G 15, 529 (1989).

[6] A. Săndulescu, A. Florescu, and W. Greiner, J. Phys. G 15, 1815 (1989).

[7] A. Florescu, A. Săndulescu, C. Cioaca, and W. Greiner, J. Phys. G 19, 669 (1993).

[8] P. Möller and J.R. Nix, At. Data Nucl. Data Tables 39, 281 (1988).

[9] A. Florescu, A. Săndulescu, and W. Greiner, J. Phys. G 19, 1947 (1993).

[10] J.R. Huizenga, H.K. Vonach, A.A. Katsanos, A.J. Gorski, and C.J. Stephan, Phys. Rev. 182, 1149 (1969).

[11] W. Dilg, W. Schantl, H. Vonach, and M. Uhl, Nucl. Phys.
A217, 269 (1973).

[12] C.H. Johnson, Phys. Rev. C 16, 2238 (1977).

[13] M. Avrigeanu, M. Ivascu, and V. Avrigeanu, Z. Phys. A 335, 299 (1990).

[14] M.K. Grossjean and H. Feldmeier, Nucl. Phys. A444, 113 (1985).

[15] J.H. Hamilton (private communication).

[16] J.H. Hamilton, A.V. Ramayya, J. Kormicki, W.-C. Ma, Q. Lu, D. Shi, J.K. Deng, S.J. Zhu, A. Săndulescu, W. Greiner, G.M. Ter-Akopian, Yu.Ts. Oganessian, G.S. Popeko, A.V. Daniel, J. Kliman, V. Polhorsky, M. Morhac, J.D. Cole, R. Aryaeinejad, I.Y. Lee, N.R. Johnson, and F.K. McGowan, J. Phys. G 20, L85 (1994).

[17] S.F. Mughabghab, M. Divadeenam, and N.E. Holden, Neutron Cross Sections (Academic Press, New York, 1981), Vol. 1.

[18] A.H. Wapstra and G. Audi, Nucl. Phys. A432, 55 (1985).

[19] L.P. Ekström and J. Lyttkens, Nucl. Data Sheets 67, 579 (1992).

[20] C.M. Baglin, Nucl. Data Sheets 66, 347 (1992). 